\title{
RUÍDOS NA UNIDADE NEONATAL: IDENTIFICANDO O PROBLEMA E PROPONDO SOLUÇÕES*
}

\author{
Márcia Maria Jordão ${ }^{1}$, Roberta Costa ${ }^{2}$, Simone Vidal Santos ${ }^{1}$, Melissa Orlandi Honório Locks ${ }^{2}$, Luciana \\ Ferreira Cardoso Assuiti ${ }^{3}$, Margarete Maria de Lima ${ }^{2}$
}

\begin{abstract}
RESUMO: Objetivou-se mensurar os ruídos e construir, junto com a equipe de enfermagem de uma unidade neonatal, estratégias para a redução dos níveis de ruídos produzidos no ambiente de trabalho. Pesquisa qualitativa, exploratório descritiva, realizada em outubro e novembro de 2016, em um hospital do sul do Brasil. Foi realizada a mensuração dos ruídos na unidade neonatal e rodas de conversa com 19 profissionais da enfermagem. Os dados foram analisados a partir da análise de conteúdo de Bardin, à luz da Política de atenção humanizada ao recém-nascido. O nível de ruídos variou de 53 a 75 decibéis e os participantes identificaram o ruído como um problema, apontando seus malefícios, levantando suas causas e propondo intervenções no ambiente e nas ações do profissional. Este estudo confirma que processos educativos promovem a transformação da realidade, garantindo o envolvimento da equipe na busca de soluções para o problema.
\end{abstract}

DESCRITORES: Ruído; Terapia intensiva neonatal; Cuidados de enfermagem; Recém-nascido.

\section{NOISE IN THE NEONATAL UNIT: IDENTIFYING THE PROBLEM AND PROPOSING SOLUTIONS}

\begin{abstract}
The aim of this study was to measure the noise and to construct, together with the nursing team of a neonatal unit, strategies for reducing levels of noise produced in the work environment. This qualitative, exploratory descriptive study was carried out in October and November of 2016, in a hospital in the south of Brazil. Measurements of the noise in the neonatal unit and conversation circles were carried out with 19 nursing professionals. The data were analyzed according to the content analysis of Bardin, considering the policy of humanized care to the newborn. The noise level ranged from 53 to 75 decibels and the participants identified noise as a problem, emphasizing its detriments, highlighting its causes and proposing interventions in the environment and in the actions of the professionals. This study confirms that educational processes promote the transformation of the reality, ensuring the involvement of the team in the search for solutions to the problem.
\end{abstract}

DESCRIPTORS: Noise; Neonatal intensive care; Nursing care; Newborn.

\section{RUIDOS EN LA UNIDAD NEONATAL: IDENTIFICANDO EL PROBLEMA Y PROPONIENDO SOLUCIONES}

RESUMEN: El objetivo fue mensurar los ruidos y construir, junto con el equipo de enfermería de una unidad neonatal, estrategias de reducción de niveles de ruidos producidos en el ámbito de trabajo. Investigación cualitativa, exploratorio-descriptiva, realizada en octubre y noviembre de 2016 en hospital del Sur de Brasil. Se efectuó medición de los ruidos en la unidad neonatal, y rondas de conversación con 19 profesionales de enfermería. Datos analizados por análisis de contenido de Bardin, a la luz de la Política de atención humanizada al recién nacido. El nivel de ruido varió entre 53 y 75 decibeles, y los participantes identificaron al ruido como un problema, señalándolo como negativo, relevando sus causas y proponiendo intervenciones en el ámbito y en las acciones del profesional. Este estudio confirma que los procesos educativos promueven la transformación de la realidad, asegurando el compromiso del equipo en la búsqueda de soluciones al problema.

DESCRIPTORES: Ruido; Cuidado Intensivo Neonatal; Atención de Enfermería; Recién Nacido. *Artigo extraído da dissertação intitulada: "Estratégias para a redução dos ruídos na unidade neonatal: uma construção
coletiva da enfermagem". Universidade Federal de Santa Catarina, 2016.

${ }^{1}$ Enfermeira. Mestre Profissional em Gestão do Cuidado em Enfermagem. Enfermeira Assistencial da unidade neonatal do Hospital Universitário da Universidade Federal de Santa Catarina. Florianópolis, SC, Brasil.

${ }^{2}$ Enfermeira. Doutora em Enfermagem. Docente de Enfermagem da Universidade Federal de Santa Catarina. Florianópolis, SC, Brasil.

${ }^{3}$ Fonoaudióloga. Doutora em Enfermagem. Fonoaudióloga da unidade neonatal do Hospital Universitário da Universidade Federal de Santa Catarina. Florianópolis, SC, Brasil. 


\section{INTRODUÇÃO}

A cada ano nascem no mundo cerca de 15 milhões de bebês pré-termo, ou seja, mais de um em cada 10 nascimentos. Muitos desses bebês que sobrevivem sofrem algum tipo de deficiência ao longo da vida, especialmente relacionadas com dificuldades de aprendizagem e problemas visuais e auditivos ${ }^{(1)}$. É certo que as modernas unidades neonatais e os avanços tecnológicos melhoraram a sobrevida destes recém-nascidos. Entretanto, estas unidades e seus aparatos tecnológicos também expõem o bebê a uma série de estímulos nocivos ao seu crescimento e desenvolvimento.

Um grave problema nas unidades neonatais é a poluição sonora. As fontes de ruído nestas unidades são as mais diversas: incubadoras, berços aquecidos, ventiladores mecânicos, bombas de infusão, monitores, alarmes, ar condicionado, manuseio de portas e gavetas, circulação e conversas de pessoas $^{(2-3)}$.

A identificação das principais fontes de ruídos em unidade neonatal faz-se necessária para evitar os potenciais danos provocados aos recém-nascidos pré-termo (RNPT) internados por longos períodos, uma vez que este local é muito diferente do ambiente intrauterino no qual o bebê estava acostumado. O bebê sai de um ambiente tranquilo, silencioso, escuro e aconchegante, para um ambiente com excesso de luminosidade e ruídos, circulação constante de pessoas e interrupções do sono e vigília, muitas vezes com desconforto e dor.

Podemos descrever como efeitos fisiológicos do excesso de ruído: aumento de pressão arterial, alterações do ritmo cardíaco, vasoconstrição periférica, dilatação das pupilas e aumento das secreções de adrenalina. Estas alterações afetam os estados fisiológico e neurocomportamental do bebê $\hat{e}^{(4)}$. A exposição ao ruído ainda gera distúrbios nos padrões de sono, irritabilidade ${ }^{(5-6)}$, agitação, choro, fadiga, aumento do consumo de oxigênio e da frequência cardíaca ${ }^{(7)}$.

Devido ao risco potencial que o ruído representa para os recém-nascidos, é necessário que os níveis sonoros presentes nas unidades neonatais sejam conhecidos, para que assim possam ser implementadas mudanças que possibilitem seu controle e redução ${ }^{(8)}$.

Cabe ressaltar que a Associação Brasileira de Normas Técnicas (ABNT) determina que os níveis sonoros confortáveis sejam de 35 a 45 decibéis $(\mathrm{dB})$. O útero oferece proteção com atenuação de até $40 \mathrm{~dB}$ de ruídos externos. Nas unidades de terapia intensiva neonatal, os níveis de ruído produzidos por alarmes, manipulação com a incubadora e conversa entre profissionais podem chegar a 77,4 dB ${ }^{(4)}$.

Diante desta problemática, desenvolvemos esta pesquisa com o objetivo de mensurar os ruídos e construir, junto com a equipe de enfermagem de uma unidade neonatal, estratégias para a redução dos níveis de ruídos produzidos no ambiente de trabalho.

\section{- MÉTODO}

Trata-se de uma pesquisa exploratória descritiva, de natureza qualitativa. O estudo foi desenvolvido na unidade neonatal de um Hospital Universitário do sul do Brasil, 100\% público, que é referência do Ministério da Saúde para o Método Canguru. A equipe de enfermagem desta unidade neonatal era composta por oito enfermeiros, 25 técnicos de enfermagem e 10 auxiliares de enfermagem.

Participaram desta pesquisa 19 profissionais da equipe de enfermagem que atuavam junto aos recémnascidos internados. Adotou-se como critério de inclusão ser lotado e atuar na unidade investigada. Foram excluídos os profissionais que se encontravam em licença saúde e maternidade, em férias, e os afastados por licença capacitação.

A coleta de dados ocorreu no período de outubro a novembro de 2016, com três estratégias: mensuração dos ruídos na unidade, rodas de conversa e questionário sobre ruídos na unidade neonatal.

A mensuração dos ruídos foi realizada nos três turnos de trabalho, sendo manhã, tarde e noite, em dias alternados. Foram aferidos os dB durante uma semana, nos ambientes da unidade neonatal, como: corredores, sala de reunião, sala de prescrição, sala de medicação, unidade de terapia intensiva neonatal 
(UTIN), incluindo dentro das incubadoras com recém-nascidos, unidade de cuidados intermediários convencional e unidade de cuidados intermediários canguru. Foi utilizado um decibelímetro da marca ICEL Manaus, modelo DL-1000, medidor de nível sonoro utilizado pela fonoaudiologia. O tempo de verificação variou de 5 a 10 minutos em cada momento e local.

Foi também utilizado um diário de campo para registro como: rotinas dos cuidados existentes nos diferentes períodos do dia, passagem de plantão, realização de exames e procedimentos, visita médica, dos pais e familiares, atendimento aos pais e discussão clínica.

As rodas de conversas também foram desenvolvidas nos três turnos de trabalho, com o objetivo de envolver o maior número de profissionais. Estas foram conduzidas pela pesquisadora principal, iniciadas com uma exposição dialogada, quando foram apresentados os registros realizados na etapa de mensuração dos ruídos e também uma síntese de estudos que foram publicados sobre a temática, com o objetivo de fomentar a discussão sobre o ambiente sonoro da unidade neonatal e identificar estratégias para redução dos ruídos.

O tempo de duração das rodas de conversa variou entre 26 e 44 minutos, sendo realizadas na sala de reuniões, dentro da própria unidade. As mesmas foram gravadas com a autorização dos participantes. Ao final de cada roda, os participantes foram convidados a preencher um questionário com questões sobre a experiência profissional e sugestões para melhoria do ambiente de trabalho, sendo que 11 questionários retornaram.

Este questionário foi utilizado como uma estratégia complementar de dados e com o objetivo de que todos os participantes pudessem expressar suas opiniões, mesmo os que não tenham feito no momento da roda de conversa.

Para análise dos resultados, foi utilizada a análise de conteúdo conforme proposta de Bardin ${ }^{(9)}$ : préanálise, exploração do material ou codificação, e tratamento dos resultados. A pré-análise contou com a seleção e organização dos dados brutos, foram utilizadas as transcrições das rodas de conversa, os registros do diário de campo e as respostas dos questionários. Na etapa de exploração do material, os dados brutos foram transformados sistematicamente em unidades menores, para possibilitar a descrição dos resultados conforme o objetivo proposto.

Nesta etapa, inicialmente, foi realizada leitura horizontal dos dados em seu conjunto, buscando as aproximações entre os depoimentos. Em seguida, fez-se a análise temática que foi dividir o texto por aproximação e similaridade em temas principais (categorias) para responder aos objetivos do estudo.

Na última etapa, foi realizado o tratamento dos resultados, buscando relacionar os dados encontrados na investigação com a literatura científica e a política de Atenção humanizada ao recém-nascido de baixo peso ${ }^{(10-11)}$.

O desenvolvimento da pesquisa foi aprovado pelo Comitê de Ética em Pesquisa com Seres Humanos da Instituição investigada com parecer número 1.721.206. Para garantir o anonimato dos participantes foram identificados nas respostas do questionário pela letra " $\mathrm{P}$ " (profissional) seguido do número sequencial, por exemplo P1, P2, P3, os depoimentos das rodas de conversa pela letra "R" com sequencial R1, R2, R3, R4.

\section{RESULTADOS}

Participaram deste estudo: cinco enfermeiras, 12 técnicos de enfermagem e dois auxiliares de enfermagem. O tempo de atuação na unidade variou de dois anos a mais de 20 anos. Em relação à mensuração dos ruídos, constatou-se que os níveis de decibéis variaram de 53 a $75 \mathrm{~dB}$, sendo que os principais causadores de ruído foram: conversas e risadas dos profissionais ( $73 \mathrm{~dB}-75 \mathrm{~dB}$ ), rede de gases (ar comprimido/oxigênio) (70 dB), alarme de bomba de seringa ( $70 \mathrm{~dB})$, dentro da incubadora sem procedimento $(64 \mathrm{~dB})$, realizando o procedimento junto com os pais $(63,6 \mathrm{~dB})$, barulho do transporte de berço (56,8 dB), abertura de armários, lixeiras e batidas de portas (53 dB).

Os dados provenientes das rodas de conversa e dos questionários foram agrupados em três categorias, com o objetivo de identificar estratégias para redução no ruído na UTIN: Reconhecendo o 
problema, Identificando as causas do problema, e Construindo soluções para o problema.

\section{Reconhecendo o problema}

Nesta categoria percebemos que os participantes reconhecem que o excesso de ruído é um problema na unidade e que acarreta inúmeros prejuízos para o recém-nascido e sua família.

Ruídos são barulhos que prejudicam o RN e o profissional e que poderiam ser evitados. O excesso de barulho pode interferir na produção láctea da mãe (R4)

Prejudicam seu quadro clínico, afetam sua estabilidade hemodinâmica, fica inquieto, agitado. Interfere no sono, queda de saturação, agitação. (P3)

Estresse, aumento do consumo de energia, aumento da produção de cortisol, hiperglicemia, aumento da frequência respiratória, quedas de saturação do oxigênio, apneia e até mesmo hemorragia intracraniana. (P1)

Desorganização, alterações cardiovasculares, prejuízos neurológicos, muitas vezes irreversíveis. (P6)

Os profissionais sentiram-se incomodados com o excesso de ruídos e perceberam que eles mesmos também podem estar sendo prejudicados com este ambiente sonoro, sendo assim motivados a buscar as fontes que podem estar causando este excesso de estímulo.

A energia muda, ficamos mais inquietos, reflete no cuidado. (R19)

Sei que o ruído atrapalha o trabalho, pode levar a falta de concentração. (R10)

Provoca desconcentração, irritabilidade, fadiga, stress e age sobre o sistema nervoso e cardiovascular do trabalhador e paciente. (P12)

[...] são estressores ambientais tanto para RN e família como para servidores. (R13)

\section{Identificando as causas do problema}

Ao reconhecer que o excesso de ruído é ruim na unidade neonatal, os profissionais conseguem identificar inúmeros fatores responsáveis por causar esta poluição sonora.

As conversas, principalmente entre os profissionais, os alarmes de equipamentos (incubadoras, bomba de infusão, monitores, etc.), o uso das torneiras (água batendo na cuba da pia), o arrastar de equipamentos, a abertura da lixeira e do hamper, tudo isso produz ruídos excessivos. (R3)

Na sala da UTI tinha tenda de oxigênio com muito barulho. (R17)

Sei pouco sobre normas e parâmetros sobre ruídos. (P7)

Quando percebemos os mesmos nos chama atenção, tentamos diminuir as conversas paralelas o tom da voz, atender logo os aparelhos e silenciar os alarmes. (P9)

Sabemos os que causam, tentamos evitar, mas de forma isolada e pontual. (R5)

Nos depoimentos dos profissionais, destaca-se o fato de que o próprio profissional de saúde é o maior responsável pelo excesso de ruído, seguido pelos equipamentos.

\section{Construindo soluções para o problema}

Ao refletir sobre o problema e identificar suas causas, os profissionais participantes foram motivados a pensar em hipóteses para melhoria da ambiência sonora da unidade.

Medir ruídos, mostrar em oficinas, conscientização. Auto regulação pessoal, com colegas com o grupo. (R3) 
Conversas mais cautelosas, ter menos manuseio com RN, agrupar os cuidados. (R13)

Melhorar os mobiliários. Cuidados com manipulação de gavetas, mais silêncio, diminuir as conversas. Passagem de plantão médica em outro ambiente. Tentar se policiar para manter iluminação e ruídos adequados. (P4)

Mensurar ruídos com frequência. Rever o ar condicionado. Mudar lixeiras, hora do soninho e psiu, sensibilizar a equipe e barulhos do computador. (P3)

Reforçar para os colegas na passagem do plantão a importância do silêncio para os nossos pacientes. Orientar pais e acompanhantes sobre esse assunto e dar exemplo. (P6)

Durante este estudo os profissionais propuseram estratégias simples para redução dos ruídos.

Eleger uma pessoa da equipe para monitorar os ruídos. Manter mais silêncio no ambiente, atentar aos alarmes. (P2)

Sinalizadores de ruídos, cartazes ou aparelhos que sinalize cores quando os decibéis acima do normal [...] lixeiras fazem muito barulho, quem sabe colocar uma borracha para diminuir o impacto. (P9)

Retomar ações simples: diminuir as luzes para a hora do soninho, placas pedindo silêncio de biblioteca, consciência profissional, porta automatizada, painel eletrônico indicando decibéis nos ambientes. (P10)

É possível perceber o engajamento, a sensibilidade, o conhecimento e o desejo de mudança desta equipe. Esta investigação possibilitou refletir sobre suas práticas e perceber que, muitas vezes, estão imersos nas rotinas diárias sem perceber os ruídos que produzem, causando angústia e vontade de melhorar, mas talvez lhes falte estímulo e mobilização.

\section{DISCUSSÃO}

A formação neurológica começa no início da vida do feto, porém em muitos aspectos o desenvolvimento é longo e demorado. Quanto mais prematuro for o nascimento do bebê, menor o seu desenvolvimento e, assim, maiores os riscos de ter complicações e sequelas. Desta forma, quando o neonato é pré-termo, o desenvolvimento cerebral, que estava acontecendo dentro da barriga da mãe, sofre alterações de acordo com o estímulo que o bebê vai receber após o nascimento ${ }^{(10)}$.

As respostas do feto aos sons já podem ser percebidas na vigésima quinta semana de vida fetal. O útero oferece proteção de até 40 decibéis $(\mathrm{dB})$ aos ruídos externos. Nas unidades neonatais, os níveis de ruídos produzidos chegam a $77,4 \mathrm{~dB}$, podendo atingir picos de $85,8 \mathrm{~dB}^{(4)}$. A mensuração realizada na unidade investigada também apontou níveis bem aquém do recomendado, entre 53 a $78 \mathrm{~dB}$.

Ruídos são aqueles sons que ultrapassam os limites estabelecidos e podem causar desconfortos e influenciar na segurança dos indivíduos, além de causar traumas auditivos e, no caso dos pré-termos, lesões neuropsicomotoras decorrentes da exposição a estes estímulos sonoros excessivos.

Um ponto bastante importante desta pesquisa se relaciona ao reconhecimento dos profissionais acerca do problema, sabendo identificar as causas, o que torna mais fácil construir estratégias de solução. Ter consciência de que os ruídos causam prejuízos, não só para recém-nascido e sua família, como também para o profissional, é o primeiro passo para modificar a realidade. A transformação da realidade é um processo lento e que requer estímulos diários para que se concretize ${ }^{(12)}$.

As hipóteses de solução elaboradas pelo grupo vêm sendo planejadas e, de forma parcimoniosa, estão sendo aplicadas à realidade, no ritmo que o próprio grupo determina. No cotidiano do cuidado, foi possível perceber a preocupação dos profissionais no sentido de garantir períodos de silêncio na unidade, estimulando conversas mais baixas. Também foram acopladas nas lixeiras da unidade pedaços de tecido, para reduzir o barulho durante seu fechamento.

Sabemos que este é um processo contínuo, que exige envolvimento dos profissionais e que deve ser revitalizado a cada dia ${ }^{(10)}$. Assim, os profissionais são impulsionados a modificar certas atitudes, no sentido de reduzir os estímulos comportamentais e ambientais. 
Os registros dos questionários e relatos nas rodas de conversa evidenciam que os profissionais reconhecem que a unidade apresenta níveis acima do ideal e que isto traz repercussões importantes para a própria equipe, podendo levar a erros na assistência. A maioria dos participantes relatou com propriedade as repercussões que os ruídos podem ocasionar, indicando que os mesmos apresentam conhecimento sobre a temática e demonstrando o reflexo dos trabalhos educativos realizados nesta unidade.

A literatura aponta que os prejuízos para os recém-nascidos são diversos, tanto imediatos como em longo prazo. Os prejuízos imediatos ocasionam alterações hemodinâmicas e interferem na sua recuperação. De forma mais tardia, a hospitalização na unidade neonatal se manifesta como: dificuldades para ouvir, pensar, conversar, ler, escrever, soletrar ou calcular, afetando o desenvolvimento social, emocional, intelectual e linguístico da criança $^{(13)}$.

Por outro lado, os profissionais de saúde, ao desenvolverem suas atividades em ambientes com níveis de pressão sonora elevados, apresentam alterações orgânicas e psicossociais, como: aumento da pressão arterial, alteração no ritmo cardíaco e no tônus muscular, cefaleia, perda auditiva, confusão, baixo poder de concentração, irritabilidade, burnout e insatisfação com o trabalho. Esta situação pode afetar a concentração do profissional, fator essencial para tomada de decisões rápidas, comum em atividades em unidade neonatais e com pacientes em estado crítico, podendo levar ao erro e comprometendo a segurança do paciente ${ }^{(14)}$.

Os profissionais participantes desta investigação sentiram-se impulsionadas a elencar estratégias para minimizar o problema, tais como medir ruídos, trabalho em equipe, termômetro de som, envolvimento dos pais e melhoria do mobiliário. Surgiu a necessidade de melhorias do serviço e também de continuar os trabalhos com a equipe para um maior crescimento profissional e aprimoramento das assistências aos pré-termos internados na unidade neonatal.

Cabe destacar que o Ministério da Saúde vem desenvolvendo e recomendando políticas para o cuidado nas unidades neonatais, visando adequações nos serviços dentro dos ambientes e nas práticas assistenciais. Dentre estas recomendações estão incluídas as condutas de diminuição dos ruídos, como: adoção de mobiliários e equipamentos com uma menor produção de ruídos; modificações das rotinas que podem provocar ruídos como mudanças nas rotinas de estudos de casos e passagem de plantão; inclusão de momentos de silêncio e utilização de estratégias para a participação das famílias ${ }^{(10)}$. Estas medidas também foram apontadas pelos participantes deste estudo.

Ao desenvolver esta investigação foi possível comprovar que a educação é uma estratégia relevante e deve permear toda a prática assistencial ${ }^{(15)}$. É através da relação dialógica que a equipe de saúde compartilha suas crenças, valores, conhecimentos e experiências, promovendo uma reflexão crítica da realidade e possibilitando a transformação de suas práticas diárias ${ }^{(16)}$.

O estudo propiciou a compreensão de que o profissional deve ser estimulado a utilizar suas habilidades e seus próprios conhecimentos para encontrar soluções para os problemas vivenciados em sua prática. Destacando-se como estratégias construídas coletivamente com equipe enfermagem da unidade neonatal investigada: Diminuir as conversas; Quando conversar, falar baixo e próximo à pessoa com está falando; Pedir às pessoas que falem baixo; Atender os alarmes prontamente; Manusear os equipamentos cuidadosamente; e Solicitar manutenção periódicas dos equipamentos e mobiliários.

Ainda, abrir/fechar as portas da unidade com cuidado; Abrir/fechar a portinhola da incubadora; Não apoiar objetos na incubadora; Não usar salto alto e evitar o uso de celular; Reduzir a campainha do telefone; Agrupar os cuidados; Realizar a hora do soninho; Passagem de plantão em sala separada das salas de internação; Colocar adesivos anti-impacto nas lixeiras, portas, gavetas e armários; Medir ruídos periodicamente; Usar termômetro de ruídos; Sinalizar a unidade com cartazes estimulando o silêncio; Manter programas educativos sobre ruídos para equipe de saúde; e Estabelecer normas de redução de ruídos - metas.

A implementação de estratégias para minimizar os níveis de ruídos nas UTIN tem como peçachave o enfermeiro, porém requer o esforço de toda equipe multiprofissional, com a participação dos gestores das instituições. Antes de esperar comportamentos adequados dos profissionais, os dirigentes devem, eles próprios, ter a clareza de que suas atitudes serão referências importantes para a equipe. As atitudes e normas subjetivas do comportamento das pessoas devem ser valorizadas, pois 
fortalecem o processo e a manutenção dos programas educativos.

Cabe destacar que os programas educativos devem vislumbrar em longo prazo as modificações comportamentais, pois estas são mais efetivas nas intervenções do meio ambiente físico, sobretudo de conscientização dos seres envolvidos no processo e sua responsabilidade em manter um ambiente acústico saudável ${ }^{(7,17)}$.

As estratégias de solução formuladas neste estudo refletem o resultado do processo reflexivo, sendo construídas coletivamente pela equipe de enfermagem, possibilitando sua aplicação na realidade com consequente transformação das práticas assistenciais.

Como limitações deste estudo, apontamos o fato desta pesquisa envolver apenas a equipe de enfermagem, entendendo que este foi o primeiro passo e que esta sensibilização deve ser estendida a toda equipe de saúde com vista a contribuir efetivamente para redução do ruído na unidade neonatal.

\section{CONSIDERAÇÕES FINAIS}

As unidades neonatais são ambientes que possibilitam a redução da morbimortalidade neonatal, os profissionais atuantes nestas unidades têm desenvolvido diversas ações no sentido de melhorar as condições do ambiente e possibilitar a qualidade de vida do recém-nascido e de sua família.

Os participantes desta investigação reconhecem que o excesso de ruído nas unidades neonatais pode trazer prejuízos para o desenvolvimento do recém-nascido, mas também para o processo de trabalho dos profissionais. De igual forma, foi possível pontuar as diversas causas do excesso de ruído neste ambiente e identificar inúmeras estratégias que podem ser empreendidas no dia a dia do cuidado para minimizar este problema.

Assim, é fundamental a manutenção de espaços democráticos, onde as questões do cotidiano possam ser melhor debatidas. Para que isso ocorra, é preciso ampliar as discussões e informações nos espaços de trabalho, permitindo e provocando a participação de todos os envolvidos, para que os trabalhadores não fiquem à margem das discussões.

Sugere-se que novas pesquisas sejam realizadas após a implementação destas estratégias para redução do ruído na unidade, com o intuito de avaliar seu impacto. Também são necessários estudos para desenvolvimento de produtos e novas tecnologias que alertem os profissionais e familiares em relação aos níveis elevados de decibéis no ambiente da unidade neonatal.

\section{REFERÊNCIAS}

1. World Health Organization (WHO). Born too soon: the global action report on preterm birth. Geneva: WHO; 2012.

2. Peixoto PV, de Araújo MAN, Kakehashi TY, Pinheiro EM. Nível de pressão sonora em Unidade de Terapia Intensiva Neonatal. Rev. esc. enferm USP. [Internet] 2011;45(6) [acesso em 29 set 2015]. Disponível: http://dx.doi. org/10.1590/S0080-62342011000600005.

3. Nazário AP, Santos VCBJ, Rossetto EG, de Souza SNDH, Amorim NEZ, Scochi CGS. Avaliação dos ruídos em uma unidade neonatal de um hospital universitário. Semina: Ciências Biológicas e da Saúde. [Internet] 2015;36(Suppl 1) [acesso em 20 ago 2016]. Disponível: http://dx.doi.org/10.5433/1679-0367.2015v36n1Suplp189.

4. Cardoso SMS, Kozlowski LC, de Lacerda ABM, Marques JM, Ribas A. Newbornphysiological responses tonoise in the neonatal unit. Braz. j. otorhinolaryngol. [Internet] 2015;81(6) [acesso em 04 out 2017]. Disponível: http:// dx.doi.org/10.1016/j.bjorl.2014.11.008.

5. Saldaña DMA, Reyes AD, Berrío MR. El ruido y lasactividades de enfermería: factores perturbadores delsueño. Investig. Enferm. Imagen Desarr. [Internet] 2013;15(1) [acesso em 18 set 2015]. Disponível: http://revistas.javeriana. edu.co/index.php/imagenydesarrollo/article/viewFile/6025/4868. 
6. Colombo G, De Bon G. Strategies to protect sleep. J Matern Fetal Neonatal Med. [Internet] 2011;24(Suppl 1) [acesso em 18 set 2015]. Disponível: http://dx.doi.org/10.3109/14767058.2011.607571.

7. Weich TM, Ourique AC, Tochetto TM, de Franceschi CM. Eficácia de um programa para a redução de ruídos em unidade de terapia intensiva neonatal. Rev. bras. ter. intensiva. [Internet] 2011;3(3) [acesso em 13 set 2015]. Disponível: http://dx.doi.org/10.1590/S0103-507X2011000300011.

8. Nogueira MFH, Ramos EG, Peixoto MVM. Identificação de fontes de ruído e de pressão sonora em unidade neonatal. Rev. enferm. UERJ. [Internet] 2011;19(4) [acesso em 10 set 2015]. Disponível: http://www.facenf.uerj.br/ v19n4/v19n4a02.pdf.

9. Bardin L. Análise de Conteúdo. Lisboa: Edições 70; 2011.

10. Ministério da Saúde (BR). Secretaria de Políticas de Saúde. Área de Saúde da Criança. Atenção humanizada ao recém-nascido de baixo peso - Método Canguru: manual do curso. Brasília: Ministério da Saúde; 2014.

11. Oliveira DC. Análise de Conteúdo temático-categorial: uma técnica maior nas pesquisas qualitativas. In: Lacerda MR, Costenaro RGS, organizadores. Metodologia da Pesquisa para a Enfermagem e Saúde: da teoria a prática. $1^{\text {a }}$ ed. Porto Alegre: Moná; 2016. p.274-89.

12. Costa R, Monticelli M. Método Mãe-Canguru sob o olhar problematizador de uma equipe neonatal. Rev. bras. enferm. [Internet] 2006;59(4) [acesso em 10 set 2015]. Disponível: http://dx.doi.org/10.1590/S003471672006000400021.

13. Daniele D, Pinheiro EM, Kakehashi TY, Balieiro MMFG. Conhecimento e percepção dos profissionais a respeito do ruído na unidade neonatal. Rev. esc. enferm. USP. [Internet] 2012;46(5) [acesso em 10 ago 2015]. Disponível: http://dx.doi.org/10.1590/S0080-62342012000500002.

14. Hassanain SM, El Raggal NM, Shalaby AA. Neonatal nursery noise: practice-based learning and improvement. J Matern Fetal Neonatal Med. [Internet] 2013;26(4) [acesso em 10 ago 2015]. Disponível: http://dx.doi.org/10.310 9/14767058.2012.733759.

15. Soares ACO, Caminha MFC, Coutinho ACFP, Ventura CMU. Dor em Unidade Neonatal: Conhecimento, Atitude e Prática da Equipe de Enfermagem. Cogitare Enferm. [Internet] 2016;21(2) [acesso em 18 dez 2016]. Disponível: http://dx.doi.org/10.5380/ce.v21i2.42897.

16. Müller EB, Zampieri MFM. Educative practice with nurses, with a view to humanized care for the newborn in the obstetric center. Texto Contexto Enferm. [Internet] 2014;23(3) [acesso em 20 set 2016]. Disponível: http:// dx.doi.org/10.1590/0104-07072014002250013.

17. da Silva CT, Terra MG, Kruse MHL, Camponogara S, Xavier MS. Multi-professional residency as an intercessor for continuing education in health. Texto Contexto Enferm. [Internet] 2016;25(1) [acesso em 10 nov 2016]. Disponível: http://dx.doi.org/10.1590/0104-0707201600002760014. 